\title{
Erratum
}

\section{Early Experience of Performing a Modified Kugel Hernia Repair with Local Anesthesia}

Junsheng Li, Yanan Zhang, Haolin Hu, and Whenhao Tang

Department of General Surgery, Affiliated Zhong-Da Hospital, Southeast University, Nanjing 210009, Jiangsu Province, P.R. China

Vol. 38: 603-608, 2008

Errors appeared in the article cited above and are corrected as follows:

- The clinical data were collected between June 2005 and April 2007.

- Eighteen of the reported cases were performed under epidermal anesthesia, not local anesthesia.

The authors sincerely apologize for the errors. 